\title{
Topology Preserving Tetrahedral Decomposition of Trilinear Cell
}

\author{
Bong-Soo Sohn \\ Department of Computer Engineering, Kyungpook National University \\ Daegu 702-701, South Korea \\ bongbong@knu.ac.kr \\ http: //bh.knu.ac.kr/ ?bongbong
}

\begin{abstract}
We describe a method to decompose a cube with trilinear interpolation into a set of tetrahedra with linear interpolation, where isosurface topology is preserved during decomposition for all isovalues. This method is useful for converting from a rectilinear grid into a tetrahedral grid in scalar data with topological correctness. We apply our method to topologically and geometrically accurate isosurface extraction.
\end{abstract}

Keywords: volume visualization, isosurface, subdivision, topology.

\section{Introduction}

Scientific simulations and measurements often generate a real-valued volumetric data in the form of function values sampled on a three dimensional (3D) rectilinear grid. Trilinear interpolation is a common way to define a function inside each cell of the grid. It is computationally simple and provides good estimation of a function between sampled points in practice. Isosurface extraction is one of the most common techniques for visualizing the volumetric data. An isosurface is a level set surface defined as $I(w)=$ $\{(x, y, z) \mid F(x, y, z)=w\}$ where $F$ is a function defined from the data and $w$ is an isovalue. The isosurface $I$ is often polygonized for modeling and rendering purposes. We call $I$ a trilinear isosurface to distinguish it from a polygonized isosurface when $F$ is a trilinear function.

Although the rectilinear volumetric data is the most common form, some techniques

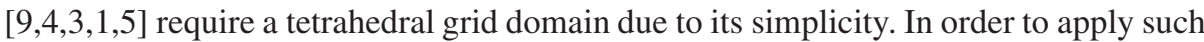
techniques to rectilinear volumetric data, people usually decompose a cube into a set of tetrahedra where a function is defined by linear interpolation. The decomposition may significantly distort the function in terms of its level sets (e.g. isosurfaces) topology and geometry. See [2] for examples. 2D/3D meshes with undesirable topology extracted from the distorted function may cause a serious inaccuracy problem in various simulations such as Boundary Element and Finite Element Method, when the extracted meshes are used as geometric domains for the simulation [10].

We describe a rule that decomposes a cube into a set of tetrahedra without changing isosurface topology for all isovalues in the cube. The rule provides topological correctness to any visualization algorithms that run on tetrahedral data converted from rectilinear data. The key idea is to add saddle points and connect them to the vertices 
of a cube to generate tetrahedra. In case there is no saddle point, we perform a standard tetrahedral decomposition method [2] without inserting any points. The tetrahedra set converted from a cube involves a minimal set of points that can correctly capture the level set topology of trilinear function because level set topology changes only at critical points. Then, we apply our method to topologically and geometrically accurate isosurface triangulation for trilinear volumetric data.

The remainder of this paper is organized as follows. In section 2, we explain trilinear isosurface and its topology determination. In section 3, we describe topology preserving tetrahedral decomposition of a trilinear cell. Then, in section 4 we give applications and results. Finally, we conclude this paper in section 5 .

\section{Trilinear Isosurface Topology}

The function inside a cube, $F^{c}$, is constructed by trilinear interpolation of values on eight vertices of the cube.

$$
\begin{aligned}
F^{c}(x, y, z) & =F_{000}(1-x)(1-y)(1-z)+F_{001}(1-x)(1-y) z \\
& +F_{010}(1-x) y(1-z)+F_{011}(1-x) y z \\
& +F_{100} x(1-y)(1-z)+F_{101} x(1-y) z \\
& +F_{110} x y(1-z)+F_{111} x y z
\end{aligned}
$$

This means the function on any face of a cell, $F^{f}$ is a bilinear function computed from four vertices on the face.

$$
F^{f}(x, y)=F_{00}(1-x)(1-y)+F_{01}(1-x) y+F_{10} x(1-y)+F_{11} x y
$$

Saddle points, where their first partial derivative for each direction is zero, play important roles in determining correct topology of a trilinear isosurface in a cube. Computing the location of face and body saddles which satisfy $F_{x}^{f}=F_{y}^{f}=0$ and $F_{x}^{c}=F_{y}^{c}=F_{z}^{c}=0$ respectively is described in [6] and [8]. Saddle points outside the cube is ignored.

Marching Cubes (MC) [7] is the most popular method to triangulate a trilinear isosurface using sign configurations of eight vertices in a cube. It is well known that some of the sign configurations have ambiguities in determining contour connectivity. The papers [68] show that additional sign configurations of face and body saddle points can disambiguate the correct topology of a trilinear isosurface. Figure 1 shows every possible isosurface connectivity of trilinear function [6].

\section{Topology Preserving Tetrahedral Decomposition}

In this section, we describe a rule for decomposing a cube with trilinear interpolation into a set of tetrahedra with linear interpolation where isosurface topology is preserved for all isovalues during the decomposition. The tetrahedral decomposition is consistent for entire rectilinear volumetric data in the sense that the tetrahedra are seamlessly matched on a face between any two adjacent cubes. The rule is based on the analysis of 


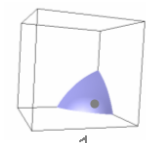

1
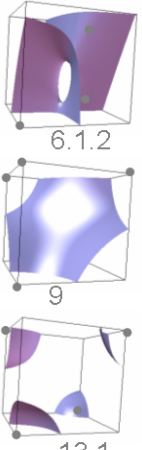

13.1

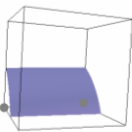

2
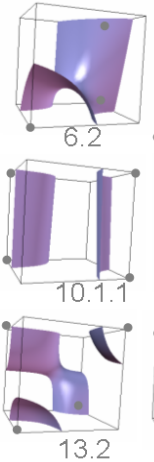
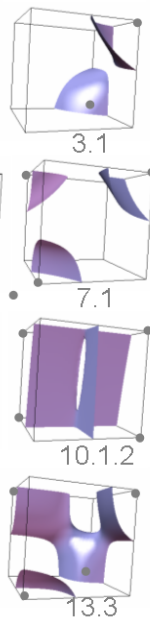

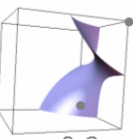

3.2
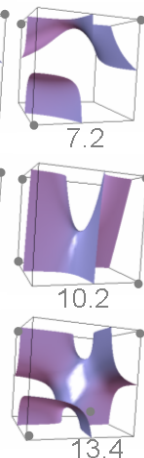

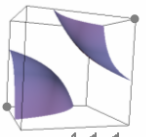

4.1.1
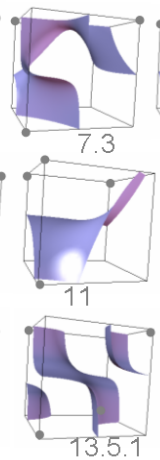
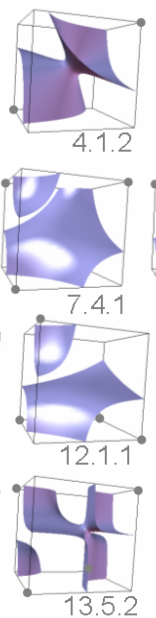
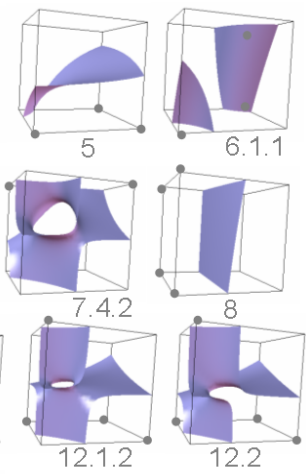

Fig. 1. Possible isosurface topology of trilinear interpolant. Numberings are taken from [6].

face and body saddles in a cell (e.g. cube). It is easy to understand the overall idea by looking at 2D case in Figure 2] which is much simpler than 3D case.

Let $s_{b}$ and $s_{f}$ be the number of body saddles and face saddles respectively. There are four cases based on the number of face saddles and body saddles, (i) $s_{b}=0$ and $s_{f}=0$, (ii) $s_{b}=0$ and $1 \leq s_{f} \leq 4$, (iii) $s_{b}=1$ and $0 \leq s_{f} \leq 4$, (iv) $s_{b}=0$ and $s_{f}=6$, and (v) $1 \leq s_{b} \leq 2$ and $s_{f}=6$. The case where the number of face saddles is six is the most complicated case that requires careful treatment. Note that the number of face saddles cannot be five. The number of body saddles cannot be two unless the number of face saddles is six. The decomposition rule for each case is as follows :

- case (i) : Decompose a cube into 6 tetrahedra without inserting any point as in [2].

- case (ii) : Choose one face saddle and decompose a cube into five pyramids by connecting the face saddle into four corner vertices of each face except the face that contains the face saddle. If a face of a pyramid contains a face saddle, the face is decomposed into four triangles that share the face saddle and the pyramid is decomposed into four tetrahedra. If a face of a pyramid does not contain a face saddle, the pyramid is decomposed into two tetrahedra in a consistent manner. If the number of face saddles is three or four, we need to choose the second biggest face saddle.

- case (iii) : Decompose a cube into six pyramids by connecting a body saddle to four corner vertices of each face of a cube. Like in (ii), if a face of a pyramid contains a face saddle, the face is decomposed into four triangles and the pyramid is decomposed into four tetrahedra. Otherwise, the pyramid is decomposed into two tetrahedra.

- case (iv) : A diamond is created by connecting the six face saddles. The diamond is decomposed into four tetrahedra. Twelve tetrahedra are created by connecting two vertices of each twelve edge of a cube and two face saddles on the two faces which share the edge. Eight tetrahedra are created by connecting each eight face of the diamond and a corresponding vertex of the cube. This will decompose a cube into twenty four tetrahedra. 


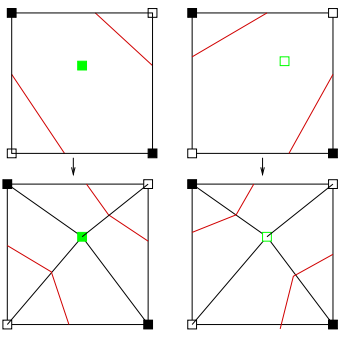

(a)

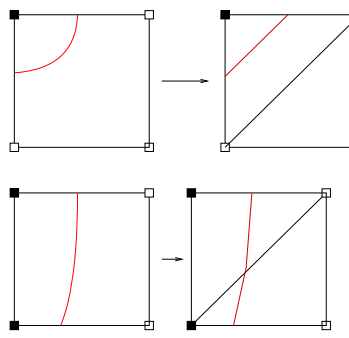

(b)
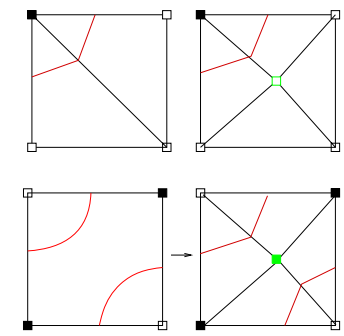

Fig. 2. (a) Triangular decomposition of a face by connecting a face saddle to each edge of the face resolves an ambiguity in determining correct contour connectivity. (b) Triangular decomposition based on a saddle point preserves level sets topology of bilinear function for every case.

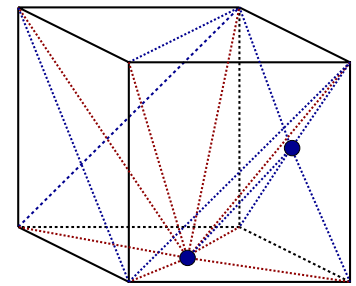

(a) $s_{b}=0, s_{f}=2$

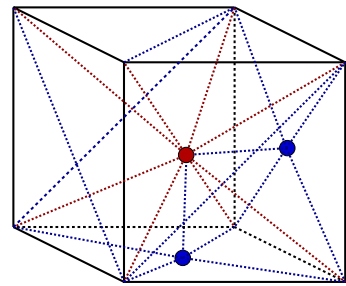

(b) $s_{b}=1, s_{f}=2$

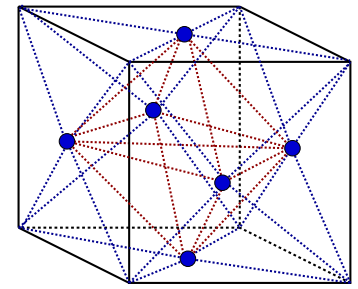

(c) $s_{b}=0, s_{f}=6$

Fig. 3. Example of rules for topology preserving tetrahedral decomposition

- case (v) : Figure 4 shows the cell decomposition when there are two body saddles and six face saddles. It generates two pyramids and four prisms where pyramids and prisms are further decomposed into tetrahedra. Choosing any two parallel faces that are connected to body saddles to form pyramids is fine. We classify saddle points as three small face saddles, a small body saddle, a big body saddle, and three big face saddles based on increasing order of the saddle values. Let small/big corner vertex be the vertex adjacent to three faces with small/big face saddles. The two parallel faces with small and big face saddles are connected to small and big body saddles respectively to form the two pyramids. The four prisms are decomposed into tetrahedra in a way that the small corner vertex should not be connected to the big body saddle and the big corner vertex should not be connected to the small body saddle. To satisfy this constraint, two types of decomposition of a prism are possible as shown in Figure 4 (c). In case $s_{b}=1$, we consider a small or big body saddle moves to a face saddle of a pyramid that is connected to the body saddle and hence the pyramid is collapsed. In this case, the pair of parallel faces for forming the pyramids are chosen in a way that the face saddle of a collapsed pyramid should not be the smallest or the biggest face saddle.

Figure 3 shows several examples of applying the tetrahedral decomposition rule to a cube with different number of body and face saddles. 


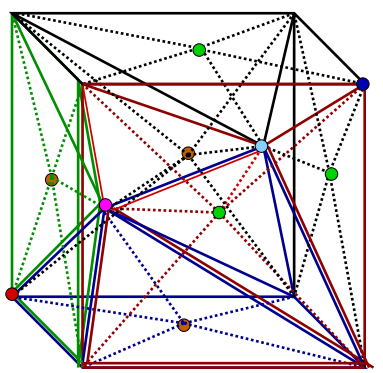

(a)

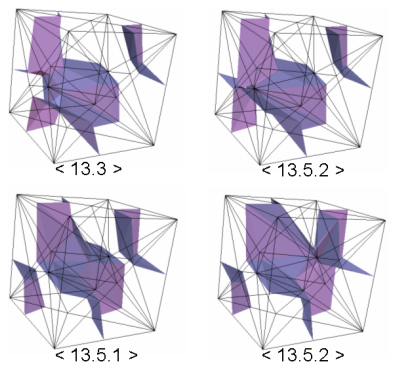

(b)

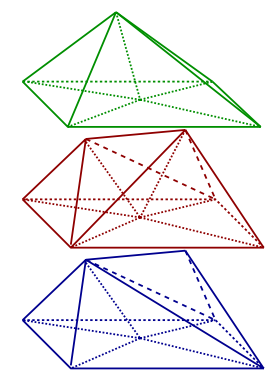

(c)

Fig. 4. (a) Cell decomposition when two body saddles exist. Dark blue, green, light blue, magenta, brown, and red circles represent small corner vertex, small face and body saddles, big body and face saddles, and big corner vertex. (b) Isosurface topology for different isovalues in case (a).

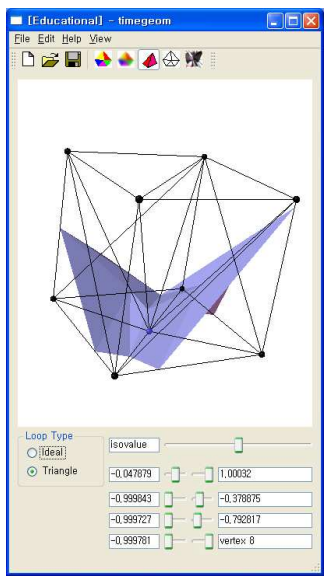

(a)

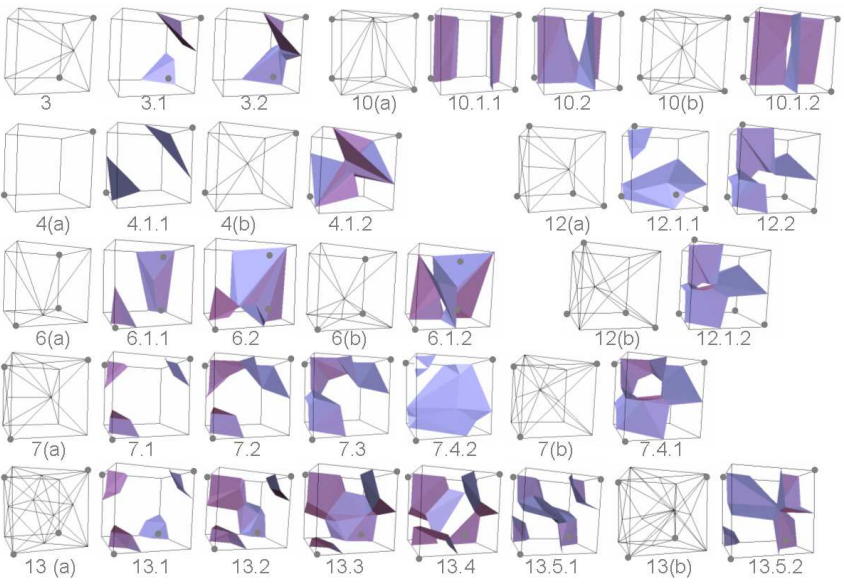

(b)

Fig. 5. (a) Demo program of topology preserving tetrahedral decomposition (b) Modified cell decomposition and triangulation for each case that has ambiguity

In appendix, we give a brief proof why topology of level sets of trilinear function in a cube is preserved during the decomposition for each case.

As shown in Figure 5(a), we implemented above method and produced a program that takes an isovalue and values of eight corner vertices of a cube, and displays tetrahedral decomposition with either a trilinear isosurface or a polygonized isosurface.

\section{Trilinear Isosurface Triangulation}

Readers may think that isosurface extraction for each tetrahedron, which is called Marching Tetrahedra (MT), after the tetrahedral decomposition would be simple and 
natural way. However, this direct application of the tetrahedral decomposition may cause extra cost in terms of the number of generated triangles and speed because even very simple case (e.g. type 1 in Figure 1) requires tetrahedral decomposition. We exploit the benefits of MC - mesh extraction with small number of elements and with high visual fidelity, and MT - ambiguity removal and simplicity by performing only necessary subdivisions.

We describe a modified cell decomposition method for resolving a triangulation ambiguity and reconstructing triangular approximation of an isosurface with correct topology. The main idea is to decompose a cube into a set of tetrahedral and pyramidal cells which do not cause a triangulation ambiguity while preserving contour topology in a cube. A facial ambiguity is resolved by decomposing a face cell into four triangles that share a face saddle point. Likewise, an internal ambiguity is resolved by decomposing a cube into six pyramids which share a body saddle point. If there is an internal ambiguity and the isosurface does not contain a tunnel shape (neck), a body saddle point is not required in the cell decomposition for reconstructing isosurface with correct topology.

There are four cases : (a) no face ambiguity with no tunnel, (b) face ambiguity (less than six) with no tunnel, (c) tunnel, and (d) six face ambiguities with no tunnel.

- case (a) : No decomposition is necessary. Just perform MC-type triangulation

- case (b) : Choose a face saddle on a face with ambiguity and decompose a cube into five pyramids by connecting the face saddle into four corner vertices of each face except the face that contains the face saddle. If a face of a pyramid contains face ambiguity, the face is decomposed into four triangles that share the face saddle and the pyramid is decomposed into four tetrahedra. If the number of face ambiguity is three, we need to choose second face saddle.

- case (c) : Decompose a cube into six pyramids by connecting a body saddle that is involved with a tunnel to four corner vertices of each face of a cube. Like in (b), if a face of a pyramid contains a face ambiguity, the face is decomposed into four triangles and the pyramid is decomposed into four tetrahedra.

- case (d) : We perform the same decomposition as the case (iv) in section 3, except that we decompose the diamond into two pyramids instead of four tetrahedra. (13(a) in Figure 5(b))

Isosurface configurations 1, 2, 5, 8, 9, and 11 in Figure 1 are the only possible cases which do not have an ambiguity in a cube. No decomposition is necessary for the cases. Triangulations for such cubes are listed in [7].

We implemented the modified decomposition method and applied it to each case in Figure 1, and extracted a triangular isosurface. Figure 5 shows the modified cell decomposition and its triangulation for every possible configuration of a trilinear isosurface that has an ambiguity. It confirms that the triangular isosurface generated from the cell decomposition is topologically equivalent to a trilinear isosurface from trilinear function by the comparison of Figure 1 and Figure 5 (b).

\section{Conclusion}

We described a method for tetrahedral decomposition of a cube where level sets topology is preserved. We envision many visualization algorithms that can take only tetrahedral 
grid data can utilize our method for dealing with trilinear volumetric data instead of using standard tetrahedral decomposition methods that may significantly distort level sets topology and geometry.

Acknowledgments. The author is grateful to Prof. Chandrajit Bajaj who gave various inspirational ideas that are related to this work. This research was supported by Kyungpook National University Research Fund, 2006.

\section{References}

1. Bajaj, C. L., Pascucci V., Schikore D. R.: The contour spectrum. In IEEE Visualization Conference (1997) 167-1737

2. Carr, H., Möller, T., Snoeyink, J.: Simplicial subdivisions and sampling artifacts. In IEEE Visualization Conference (2001) 99-108

3. Carr, H., Snoeyink, J.: Path seeds and flexible isosurfaces using topology for exploratory visualization. In Proceedings of VisSym (2003) 49-58

4. Carr, H., Snoeyink, J., Axen, U.: Computing contour trees in all dimensions. Computational Geometry: Theory and Applications Vol. 24. (2003) 75-94

5. Edelsbrunner, H., Harer, J., Natarajan, V., Pascucci, V.: Morse complexes for piecewise linear 3-manifolds. In Proceeding of the 19-th ACM Symp. on Comp. Geom. (2003) 361-370

6. Lopes, A., Brodlie, K.: Improving the robustness and accuracy of the marching cubes algorithm for isosurfacing. IEEE Transactions on Visualization and Computer Graphics (2003) $19-26$

7. Lorensen, W.E., Cline, H.E.: Marching cubes: A high resolution 3D surface construction algorithm. In ACM SIGGRAPH (1987) 163-169

8. Natarajan, B.K.: On generating topologically consistent isosurfaces from uniform samples. The Visual Computer, Vol. 11. (1994) 52-62

9. Nielson, G.M., Sung, J.: Interval volume tetrahedrization. In IEEE Visualization Conference (1997) 221-228

10. Zhang, J., Bajaj, C. L., Sohn, B.-S.: 3D finite element meshing from imaging data. Computer Methods in Applied Mechanics and Engineering (CMAME) Vol. 194. (2005) 5083-5106

\section{Appendix: Proof of Topology Preservation During Decomposition}

We call the isosurface extracted from a set of tetrahedra as PL isosurface. The proof is done by showing that, for any isovalue in a cube, the numbers of PL isosurface components and trilinear isosurface components are the same and PL isosurface components are topologically equivalent to trilinear isosurface components. Note that PL isosurface inside a cube should be always a manifold (except for a degenerate case). There is no isolated closed PL isosurface component in a cube.

First of all, it is easy to see that the decomposition preserves trilinear isosurface topology on each face. A face is decomposed into four triangles when there is a face saddle on the face and decomposed into two triangles when there is no face saddle. There are three possible contour connectivity except for the empty case where symmetric cases are ignored. Figure 2 shows that each isocontour connectivity of bilinear function on a face is preserved for any possible triangular mesh generated from our decomposition rule. 
We classify corner vertices and saddles into up-vertices and down-vertices based on the check whether a function value of a vertex is bigger than or lower than an isovalue. We use a term, component-vertices, to indicate either up-vertices or down-vertices that have bigger or same number of connected components compared to the other one. Except for the configurations 13.5.1 and 13.5.2 in Figure 11 a connected component of component-vertices uniquely represents an isosurface component. Consider cases (i), (ii), and (iii). If there is no hole, connected components of component-vertices on faces are separated each other inside a cube to form a simple sheet (disk) for each connected component, which is consistent with actual trilinear isosurface topology. If there is a hole, the connected components of component-vertices on faces are connected through a saddle point inside a cube to form a tunnel isosurface. The reason why we choose the second biggest face saddle in the case (ii) with three or four face saddles and (v) with one body saddle is to avoid connecting components of component-vertices inside a cube that needs to be separated. For example, if we choose the smallest or the biggest face saddle in the configuration 7.2, two components of component-vertices on faces of a cube can be connected through an edge and hence two separate isosurface components would be connected with a tunnel.

In cases (iv) and (v) where the number of face saddles is six, the configurations except for 13.5.1 and 13.5.2 are proved in a similar way as the cases of (i), (ii), and (iii). The configurations 13.5 .1 and 13.5 .2 can be proved by taking out tetrahedra that contributes to the small isosurface component and apply the same proof of (i), (ii), and (iii) to the rest of isosurfaces for topological correctness. 